\title{
ACTIVIDADES MULTIAVENTURA PARA EL DESARROLLO SOCIAL Y EMOCIONAL EN POBLACIÓN INFANTO-JUVENIL
}

\author{
M. P. Aparicio-Flores \\ Universidad de Alicante \\ María Vicent \\ Universidad de Alicante \\ Cándido, J. Inglés \\ Universidad Miguel Hernández de Elche \\ David Aparisi-Sierra \\ Universidad de Alicante \\ Ma Isabel Gómez-Núñez \\ Universidad Católica San Antonio de Murcia \\ Aitana Fernández-Sogorb \\ Universidad de Alicante \\ https://doi.org/10.17060/ijodaep.2017.n1.v3.972
}

Fecha de Recepción: 21 Enero 2017

Fecha de Admisión: 1 Abril 2017

\section{RESUMEN:}

La actividad física y el deporte reduce problemas de obesidad y enfermedades hipocinéticas, además de aumentar el bienestar mental y las habilidades sociales, en todo tipo de población. Sin embargo, con la llegada de la adolescencia, esta práctica decae significativamente, por varias razones entre las que se encuentran la carencia de tiempo libre, la pereza y las preocupaciones perfeccionistas. Por lo que, la institución educativa debería tomar conciencia de dicha problemática y poner en práctica programas de intervención. El punto clave es diseñar acciones colaborativas en las que intervenga el ocio y tiempo libre en un escenario natural, donde las actividades multiaventura tienen un gran papel. El objetivo de esta investigación es conocer la percepción de treinta monitores, de un campamento multiaventura situado en la provincia de Alicante, sobre el desarrollo social y emocional que consideran beneficioso para el alumnado desde las actividades que promueven las instalaciones de educación no formal donde trabajan. Se empleó una metodología cualitativa, con una entrevista semiestructurada y desde una orientación narrativa-biográfica. Los resultados reportan una percepción positiva, en la mayoría de los casos, respecto a los temas estudiados.

Palabras clave: Actividades multiaventura, desarrollo social, contribución emocional, superación personal, cooperación. 


\title{
ACTIVIDADES MULTIAVENTURA PARA EL DESARROLLO SOCIAL Y EMOCIONAL EN POBLACIÓN INFANTO-JUVENIL
}

\begin{abstract}
:
Adventure activities for social and emotional development in chil-youth population

Physical activity and sports reduce problems of obesity and hypokinetic diseases, at the same time, it increases mental well-being and social skills, in all types of population. However, with the advent of adolescence, this practice declines significantly, for several reasons among which are the lack of free time, laziness and perfectionist concerns. So, the educational institution should be alarmed of the problem and implement intervention programs. The key point is to design collaborative actions in the leisure time to intervene in a natural setting, where adventure activities have a great role. The aim of the research is to understand the perception of thirty monitors, a multisport camp located in the province of Alicante, on the social and emotional development they considered can benefit the students from the activities that promote educational not formal facilities where they work. We work from a qualitative methodology, with a semi-structured interview and from a narrative-biographical orientation. The results report a positive perception, in most cases, on all the subjects studied.
\end{abstract}

Keywords: Multi-adventure activities, social development, emotional contribution, self-improvement, cooperation.

Uno de los grandes obstáculos de la sociedad actual es el sedentarismo y más allá del mismo, las influencias que reporta sobre la propia salud, construyendo un mayor riesgo de enfermedades hipocinéticas que pueden originarse desde la infancia (Bailey, 2005). Según Mollá-Serrano (2007), España presenta un alto porcentaje de personas con carencia de actividad física, destacando, por encima de ello, la analfabetización de los posibles medios para cambiar esta situación.

Es la adolescencia la época en la que tiene lugar la mayor tasa de abandono de la actividad física y el deporte por diversos motivos, como la carencia de tiempo libre, estudios, conflicto de intereses (Burton y Martens, 1986), por aburrimiento, pereza o desgana (Mollá-Serrano, 2007), y lo más destacable, por factores estresantes como la excesiva presión ajena que conducen a la ausencia de disfrute (Cohn, 1990). Existen numerosas investigaciones que demuestran rasgos perfeccionistas desadaptativos vinculados al deporte (García-Fernández et al., 2016), contemplando que el miedo al fracaso crea en ellos una falta de diversión (Fiorese, Andrade y Lopes, 2013). Slade y Owens (1998) argumentaron que el Perfeccionismo Socialmente Prescrito (PSP) se relaciona con el miedo al fracaso, provocando la evitación de resultados negativos y el comportamiento basado en la motivación extrínseca. Sin embargo, a pesar de que el perfeccionismo ha sido considerado, generalmente, como un constructo desadaptativo, existen evidencias de algunas facetas que pueden llegar a considerarse positivas y gratas en algunos casos. Según Stoeber y Otto (2006) es importante distinguir entre los esfuerzos y las preocupaciones perfeccionistas, debido a que estas últimas son principalmente las que marcan el perfeccionismo en el deporte. Contrariamente, los esfuerzos perfeccionistas son asociados con características positivas tanto en el proceso como en el resultado (Stoeber, 2011, 2014; Stoeber, Stoll, Salmi y Tiikkaja, 2009).

Malkinson y He (2014) expresan que, como entes biológicos, los individuos necesitan del movimiento activo para el bienestar físico y emocional. Según los autores, la participación en deportes individuales o colectivos facilita la auto-comprensión y el entendimiento de otras culturas y estructuras de valores favoreciendo, consecuentemente, un enfoque integral y equilibrado (Malkinson y $\mathrm{He}, 2014)$.

Existen numerosas investigaciones que evidencian que el desarrollo de actividad física mejora la calidad de vida con el aumento de ella, reduciendo enfermedades como la diabetes, la presión 
arterial, afecciones coronarias (Bailey, 2005) y beneficios tanto físicos como sociales (Neely y Holt, 2014) en todo tipo de población (véase Smith, 2015, para una revisión). También se hallan investigaciones que exponen la actuación física como el desarrollo integral de la mente y el cuerpo, la autoconfianza y la autoestima, generando un estado de ánimo positivo y una mayor confianza en sí mismo, y contribuyendo, además, en la disminución de ansiedad, depresión, tensión y estrés; lo que beneficia psicológicamente y favorece el desarrollo de la personalidad (Bailey, 2005).

La educación al aire libre en contacto con la naturaleza puede ser una herramienta muy poderosa en la mejora de las relaciones sociales (Martin, 2008; Murillo-Castro, Muñoz-Rojas y EliondoSalazar, 2009). Zurita-Pérez (2009) manifiesta que la riqueza de sensaciones propiciada por la ocupación de ocio y tiempo libre en la naturaleza contribuye al cambio de carácter de quienes lo practican. Tal y como expresan Pryor, Carpenter y Townsend (2005) cuyos autores determinan que la educación al aire libre constituye una estrategia de intervención saludable en toda la población, tanto en el bienestar generalizado de la sociedad como en personas con vulnerabilidades identificadas. De ahí que la tarea de actividades de aventura en la naturaleza haya emergido como objeto de estudio, desde la educación, en estos últimos años.

\section{EL PRESENTE ESTUDIO}

El presente estudio trata de analizar las percepciones observadas de un grupo de profesionales que se dedican a la educación no formal de actividades multiaventura con el objetivo de comprender, desde su punto de vista técnico y competente, la contribución emocional y social que estiman poder desplegar en los jóvenes, desde sus puestos de trabajo, las instalaciones con las que cuentan y la metodología empleada para ello. En consecuencia, las cuestiones de investigación planteadas fueron las siguientes:

¿Qué percepción sobre el desarrollo social tiene el grupo de monitores profesionales acerca de la contribución de las actividades al aire libre, planificadas para el alumnado de Educación Infantil (EI), Educación Primaria (EP) y Educación Secundaria Obligatoria (ESO?

¿Qué vinculación establecen entre el desarrollo emocional del alumnado de EI, EP y ESO y las actividades multiaventura que se realizan en el campamento?

\section{MÉTODO}

\section{Descripción del contexto y de los participantes}

Se obtuvo una muestra compuesta por 31 profesionales, con edades comprendidas entre los 18 y 45 años de edad ( $M_{e d a d}=27.6$ años), trabajadores del Campamento de Fontés (CF), de los cuales un $54.84 \%$ fueron mujeres y un $45.16 \%$ varones. Fue excluido 1 participante por omisiones en sus respuestas. De manera que, la muestra definitiva quedó compuesta por 30 participante. Estas instalaciones velan por la educación infanto-juvenil de una manera divertida, lúdica y con grandes vinculaciones con la naturaleza, situado a las afueras de una pequeña localidad semiurbana perteneciente a la comarca de l'Alcoià, en el interior de la provincia de Alicante. Actividades como piragüismo, escalada, tirolina, espeleología entre gran cantidad de acciones más, son las encargadas de dinamizar la parte física de las instalaciones. La institución cuenta con una plantilla de trabajadores de, aproximadamente, iguales dimensiones que los participantes encuestados y tiene la capacidad de albergar unos 450 alumno/as por día, de entre 3 y 16 años de edad. La mayoría del alumnado que pasa por el CF pertenece a familias con un nivel socioeconómico medio; condiciones similares a los participantes de la investigación. 


\section{ACTIVIDADES MULTIAVENTURA PARA EL DESARROLLO SOCIAL Y EMOCIONAL EN POBLACIÓN INFANTO-JUVENIL}

\section{Medidas}

Una entrevista semiestructurada, contemplando las recomendaciones de Potter y Hepburn (2005) fue el instrumento elegido para la recogida de datos, considerando la investigación biográfica-narrativa como el eje fundamental para observar, con claridad, las aportaciones de los participantes desde sus propias experiencias. En la entrevista, se planteaban a los docentes diversas cuestiones sobre la influencia a nivel emocional y social, que según su opinión, ejercen las actividades desarrolladas en el campamento. Asimismo, se les pidió que valorasen dichas influencias como positivas o negativas y que justificasen su visión. Este trabajo se basó, del mismo modo, en los criterios propuestos por Connelly y Clandinin (1995) según los cuales, toda investigación narrativa ha de basarse en los criterios de claridad, verosimilitud y transferencia.

\section{Procedimiento}

Las entrevistas fueron entregadas a los monitores del campamento en papel, aprovechando una jornada de formación de la institución privada. Las cuestiones plasmadas en la entrevista fueron tratadas con los criterios de fiabilidad oportunos regidos por Goetz y Lecompte (1988). Se repartieron los documentos a los distintos empleados que respondieron de manera anónima y voluntaria, tras haber sido informados de los objetivos de la investigación (Braun, Gable y Kite, 2011).

Una vez explicado el propósito y devueltas las entrevistas cumplimentadas por los participantes, fueron transcritas de manera virtual. Asimismo, a cada una de las entrevistas se les asignó un código numérico que las identificara, para salvaguardar el anonimato.

\section{Tratamiento de datos}

Una vez recogidas todas las entrevistas se realizó un sistema de códigos demográficos e inferenciales para poder analizar las narrativas. Para ello, se realizó una primera lectura exhaustiva de todas las entrevistas, contemplando cuidadosamente todos los argumentos de los participantes, desde una interpretación objetiva, con la finalidad de extraer un mapa de códigos y sus respectivas definiciones, que diesen lugar a los posteriores datos inferenciales.

Seguidamente, se trataron todos los datos de la entrevista con el programa AQUAD versión 6, con el propósito de obtener una segunda lectura, de cada segmento codificado, comprendida por los códigos conformados.

\section{RESULTADOS}

\section{Tema 1: Desarrollo Social}

En las líneas que siguen se analizarán los resultados obtenidos sobre la percepción que tienen Ios monitores profesionales del CF en relación a la contribución sobre el desarrollo social de la persona, contemplando las actividades multiaventura que se trabajan desde esta institución privada. Dentro de esta temática se derivan dos códigos que configuran el beneficio facilitador o el reducido (véase Tabla 1).

\section{Exposición de los datos}


LA PSICOLOGÍA HOY: RETOS, LOGROS Y PERSPECTIVAS DE FUTURO. PSICOLOGÍA INFANTIL

Tabla 1.

Resultados obtenidos para los códigos pertenecientes al Tema 1. Desarrollo social

\begin{tabular}{|c|c|c|c|c|c|c|c|}
\hline Códigos & & Subcódigo & $\begin{array}{l}\text { Casos } \\
\text { con } \\
\text { hallazgo }\end{array}$ & $\begin{array}{l}\text { Presencia } \\
\text { del } \\
\text { subcódigo } \\
(\%)\end{array}$ & $\begin{array}{l}\text { Ausencia } \\
\text { del } \\
\text { subcódigo }\end{array}$ & Frecuencia & $\begin{array}{l}\mathrm{N}^{\circ} \text { de } \\
\text { participantes }\end{array}$ \\
\hline \multirow[t]{4}{*}{1.1 . } & Facilitador & 1.1.1. Heterogeneidad & 19 & $37.50 \%$ & $62.50 \%$ & 21 & 30 \\
\hline & $(94.63 \%)$ & 1.1.2. Compañerismo & 16 & $30.35 \%$ & $69.65 \%$ & 17 & 30 \\
\hline & & 1.1.3. Aprendizaje & 9 & $16.07 \%$ & $83.93 \%$ & 9 & 30 \\
\hline & & 1.1.4. Confianza & 5 & $10.71 \%$ & $89.29 \%$ & 6 & 30 \\
\hline \multirow[t]{2}{*}{1.2 . } & Reducido & 1.2.1. Falta de tiempo & 1 & $3.58 \%$ & $96.42 \%$ & 2 & 30 \\
\hline & $(5.37 \%)$ & 1.2.2. Personas conocidas & 1 & $1.79 \%$ & $98.21 \%$ & 1 & 30 \\
\hline
\end{tabular}

La percepción mayoritaria de los participantes entrevistados sobre las actividades del CF destaca intensamente sobre la idea facilitadora del desarrollo social de aquellos sujetos que las practican (véase Tabla 1).

Este facilitar su desarrollo social, los encuestados lo perciben a través de diferentes matices, recogidos como subcódigos, de diversa intensidad.

Dentro de esta tesitura, es posible visualizar con mayor prevalencia la idea de heterogeneidad, en la que se destaca la percepción de que, a través de las dinámicas existentes para hacer caer al alumnado en grupos distintos a los formados en el aula ordinaria día a día, se contribuye de una manera reflexiva en el desarrollo social del estudiantado que pasa por su centro (véase Tabla 1). Un ejemplo de ello podría visualizarse en la entrevista siguiente:

"Sí, una de las estrategias de Fontés es el trabajo con grupos heterogéneos, desde el campamento se considera que ayuda a los niños a interactuar con compañeros que suelen tener menos contacto, se separan de su grupo de amigos y se fomenta la igualdad" (009).

Por otra parte, en una gran proporción de las narrativas de los participantes se enfatiza el valor del compañerismo. Este juicio infiere en el fomento inculcado de convivencia e igualdad que promueve el campamento desde sus actividades grupales. Un ejemplo posible a seguir pueden ser las palabras expuestas por uno de los encuestados:

"En Fontés educamos en valores como el compañerismo, el respeto hacia los demás, el entorno hacia nosotros mismos, entre otros" (029).

Del mismo modo, los participantes subrayan la socialización y aprendizaje a través de la imitación de los monitores contribuyente en el desarrollo social infanto-juvenil, en un $16.07 \%$ de los resultados:

"El trabajo es vivencial y los niños aprenden con el ejemplo" (E004).

Por último, como elemento facilitador, una décima parte de los monitores entrevistados resalta la importancia del favorecimiento de seguridad que desarrolla el alumnado en sí mismo y en sus iguales, desde la idea de confianza, al necesitar la ayuda de estos últimos y al superar obstáculos encontrados en la consecución de las actividades. Un ejemplo de ello lo observamos en la narrativa siguiente:

"En las actividades a realizar los chavales tienen miedos y con ellas se van superando y uniendo" (006). 


\section{ACTIVIDADES MULTIAVENTURA PARA EL DESARROLLO SOCIAL Y EMOCIONAL EN POBLACIÓN INFANTO-JUVENIL}

Cabe considerar, con un porcentaje inferior, manifiestamente muy distante $(5.35 \%)$, se identifican dos factores que limitan la contribución al desarrollo social. En este sentido, el subcódigo que lo integra muestra la falta de tiempo, como punto negativo en la socialización de los pequeños:

"Tampoco se pueden hacer milagros en un día" (E025).

En menor presencia, se destaca el subcódigo de personas conocidas en el que, únicamente uno de los profesionales expresa la idea de no aportación social ya que pasan el día con los mismos niños del centro escolar:

"En este caso no pienso que sea de una forma increíblemente grande, puesto que el grupo con el que interaccionan es el mismo que lo hacen a diario" (E015).

\section{Tema 2: Desarrollo Emocional}

Seguidamente son expuestos los resultados obtenidos acerca del desarrollo emocional percibido por los monitores profesionales del CF desde la ejecución de actividades multiaventura, con dos códigos que intensifican o condicionan el beneficio.

Exposición de los datos

Tabla 2.

Resultados obtenidos para los códigos pertenecientes al Tema2. Desarrollo emocional

\begin{tabular}{|c|c|c|c|c|c|c|}
\hline Códigos & Subcódigos & $\begin{array}{l}\text { Casos } \\
\text { con } \\
\text { hallazgo }\end{array}$ & $\begin{array}{l}\text { Presencia } \\
\text { del } \\
\text { subcódigo } \\
(\%)\end{array}$ & $\begin{array}{l}\text { Ausencia } \\
\text { del } \\
\text { subcódigo } \\
(\%)\end{array}$ & Frecuencia & $\begin{array}{l}\mathrm{N}^{\mathrm{o}} \text { de } \\
\text { participantes }\end{array}$ \\
\hline \multirow{3}{*}{$\begin{array}{l}\text { 2.1. Intensificado } \\
(96.30 \%)\end{array}$} & 2.1.1. Superación & 19 & $38.89 \%$ & $61.11 \%$ & 21 & 30 \\
\hline & $\begin{array}{l}\text { 2.1.2. Emociones } \\
\text { compartidas }\end{array}$ & 16 & $33.33 \%$ & $66.67 \%$ & 18 & 30 \\
\hline & 2.1.3. Rol monitor & 11 & $24.08 \%$ & $75.92 \%$ & 13 & 30 \\
\hline
\end{tabular}

En el análisis de las narrativas, atendiendo a la percepción de los participantes sobre el desarrollo emocional, los encuestados destacan plenamente una intensa vinculación anímica en la práctica de las actividades multiaventura (véase Tabla 2).

Prevalece en la muestra de entrevistados la idea de superación personal de los jóvenes que practican las actividades, significando la importancia de enfatizar situaciones que los hacen creer en ellos mismos. De manera que, cuando se ejecuta una actividad que, en un principio, puede causar miedo, con ayuda de la motivación del monitor y de sus iguales, existe la posibilidad de superar ese obstáculo.

Dos de los ejemplos destacables pueden ser leídos a continuación:

"Por supuesto que influye, puesto que aquí se enfrentarán a situaciones en las que normalmente no se encuentran. Además de desarrollar ciertas destrezas, también trabajan con el miedo y cómo superarlo. La ayuda mutua o el respeto por los compañeros es fundamental. Al final de la jornada se dan cuenta que son capaces de hacer más cosas de las que piensan normalmente" (015). "Para ello expondré un ejemplo: En una jornada del año 2015, un alumno con parálisis cerebral y espasticidad muscular, empezó las actividades llorando y con impotencia y terminó superando todas las actividades, provocando en él una emoción de satisfacción y alegría" (016). 
Como subcódigo secundario (33\%), el carácter emocional compartido se enfatiza de forma acentuada, en la que los participantes aportan ideas relacionadas con nexos de unión afectivos. Patrones como los descritos a continuación son narrados por los mismos:

"Al interactuar muchos participantes en las diferentes actividades se establecen conexiones emocionales o vínculos mediante los cuales los diferentes jóvenes comparten emociones" (E024). "Es una actividad diferente a lo que son las actividades del día a día. Tiene muchas características como que se hace en un lugar de naturaleza y abierto, y esto ofrece una diversidad de conexiones consideradas buenas o malas, todo depende. Excitación, diversión, miedos, ilusión, alegría, etc. Al exponerse de cerca a estos cambios emocionales se produce una fusión que indirectamente les ayuda al autoconocimiento" (E010).

Por último, cabe resaltar el rol del monitor, en el que los participantes subrayan la relevancia de la implicación del monitor hacia el desarrollo anímico de los jóvenes que pasan por el campamento:

"El campamento da las herramientas y las situaciones para que se produzcan, pero el monitor debe saber gestionarlas y potenciarlas" (E015).

Sin embargo, el estudio, aunque con un porcentaje muy alejado (3.70\%), también admite el condicionamiento que coarta el desarrollo emocional de los jóvenes en la ejecución de actividades multiaventura (véase Tabla 2). Esta idea dota de referencia a la tristeza percibida en el alumnado cuando no consigue superar las pruebas:

"Hay aspectos internos y externos. Siendo un excelente monitor puede producirse una gran frustración o retroceso emocional en un niño por falta de tiempo para abordar una situación concreta que pueda surgir" (E004).

Es desde este tipo de narrativas donde los monitores expresan de manera abierta y oral que el hecho de limitarse a un tiempo destinado para cada actividad puede verse condicionado, y en ocasiones puntuales, el empeño estimulante que ponen los profesionales en un joven con el objetivo de conseguir superar un miedo determinado, no sea del todo eficaz.

\section{DISCUSIÓN}

En este trabajo se propuso examinar la percepción de los profesionales del CF sobre el desarrollo social y emocional de la población infanto-juvenil. Los monitores coinciden en afirmar que las actividades diseñadas desde la institución en la que trabajan, contribuyen con un alto porcentaje en el desarrollo de la persona.

En lo que respecta al desarrollo social del alumnado que pasa por su centro, los participantes afirmaron que las actividades trabajadas en el CF benefician altamente las habilidades sociales. Tal y como expresa Martin (2008) con la idea de que la educación al aire libre en contacto con la naturaleza puede ser una herramienta muy poderosa en la mejora de las relaciones sociales. Aspecto que da respuesta a nuestra primera cuestión de investigación.

De los resultados observados respecto al código que facilita el desarrollo social, se destaca la percepción, de gran parte de los participantes, en cuanto a la contribución social completada a través de las dinámicas existentes para hacer caer al alumnado en grupos heterogéneos. En esta misma línea, se sitúan investigaciones de autores como Murillo-Castro et al. (2009), quienes consideran el fortalecimiento personal y la unión grupal como resultados clave en la ejecución de actividades al aire libre.

Asimismo, casi la tercera parte de los monitores entrevistados incidieron en el beneficio inculcado de convivencia, igualdad y compañerismo que fomenta el campamento desde sus actividades grupales. Este pensamiento es vinculante a investigaciones llevadas a cabo por Greblo, Bari y Ceci 
(2016), en el que un estudio compuesto por 258 atletas adolescentes, demostró que los esfuerzos perfeccionistas de las jóvenes se relacionaron positivamente con la percepción de un clima motivador, que incitaba a la relación de apoyo, esfuerzo y mejora de la actividad, cuestión apoyada por Stoeber $(2011,2014)$. En este sentido, es importante tener en cuenta el esfuerzo de los jóvenes realizado gracias al apoyo percibido y el clima de cooperación creado desde la institución, aportando una gran motivación en el alumnado para llegar a conseguir su propósito, teniendo en cuenta que un alto PSP correlaciona positiva y significativamente con la Ansiedad escolar (Inglés, GarcíaFernández, Vicent, Gonzálvez y Sanmartín (2016) pero también con la Apertura a la experiencia (Vicent, Inglés, Gonzálvez, Sanmartín y García-Fernández, 2016).

En otro orden de ideas, y aún desde el desarrollo social que los monitores pueden percibir, un pequeño colectivo del CF, expresó que aunque la actividad física contribuye al desarrollo social del alumnado, el tiempo limitado es un punto negativo a destacar en la socialización de los pequeños. Zurita-Pérez (2009) señala que con una única sesión de actividad física no es posible cubrir el expediente académico anual. Sin embargo, la autora destaca la necesidad insistente de este tipo de actividades, pudiendo ser el punto de inflexión hacia la contribución de un aprendizaje significativo de calidad que reporte todo tipo de beneficios.

Por otra parte, y enlazando con el segundo tema tratado, la mayor parte de los comentarios adscritos reciben una percepción intensificada en cuanto al aumento de desarrollo emocional por parte del alumnado que realiza actividades multiaventura en el campamento. Aspecto que responde a nuestra segunda cuestión de investigación. Es destacable la percepción de superación infanto-juvenil cuando se ejecuta una actividad que, en un principio, puede causar miedo, con ayuda de la motivación del monitor y de sus iguales. Esta apreciación se encuentra en vinculación con aportaciones como las de Flett y Hewitt (2005) que analizan los peligros del perfeccionismo en el deporte, expresando que las percepciones de autocontrol y autoeficacia, gracias a un enfrentamiento, desarrollan un sentido de flexibilidad ajustado con la situación, que reorganiza niveles automáticos de funcionamiento personal, y asocia un vínculo positivo entre las acciones y el resultado de las mismas. Asimismo, Bailey (2005) declara que una actuación física, en el medio natural, desarrolla una mayor autoconfianza, liberando de ansiedades, depresiones, tensiones y estrés; aspecto beneficioso en el desarrollo psicológico y personal de los sujetos.

Del mismo modo, es relevante destacar la aportación de ideas relacionada con los beneficios a nivel emocional, tal y como exponen Pryor et al. (2005) asegurando que una intervención física al aire libre produce un bienestar generalizado en la persona, incluso en las que se identifica cierta vulnerabilidad emocional.

Por último, como método intensificador de las emociones, se observan participantes que atribuyen una gran importancia al papel motivante del monitor. Tal y como expresa Zurita-Pérez (2009) la cual afirma la necesidad de promocionar un aprendizaje significativo de calidad en el área de EF, que no englobe solamente beneficios físicos sino una contribución íntegra del individuo.

Cabe mencionar que este trabajo tiene diversas limitaciones. Estos resultados no son generalizables a otros grupos que no sean profesionales del ámbito de la educación no formal. Asimismo, estos resultados es posible que varíen empleando una metodología distinta a la cooperativa, utilizada por los profesionales del campamento.

\section{CONCLUSIONES}

En síntesis, los beneficios a nivel social y emocional de las personas practicantes de actividades multiaventura son percibidos altamente por los profesionales del CF.

Es relevante contemplar la idea de Martínez-Navarro (1993) a través de la "pedagogía del ocio"; en la que el enlace del tiempo libre con la perspectiva de educación no formal en la EF, construya un 
espacio educativo potencialmente enriquecedor, que erradique las causas de abandono de la actividad física en los jóvenes, expresadas por Cohn (1990) y Mollá-Serrano (2007) como el aburrimiento, la pereza y la desgana. De manera que se potencien las causas primordiales de inicio en la actividad físico-deportiva entre el público infanto-juvenil, en las que prime la diversión, cooperación, y el entretenimiento entre amigos, y dé como resultado un factor adaptativo para todo tipo de población incluso con vulnerabilidades o psicopatologías asociadas.

\section{REFERENCIAS BIBLIOGRÁFICAS}

Bailey, R. (2005). Evaluating the relationship between physical education, sport and social inclusión. Educational Review, 57(1), 71-90. doi:10.1080/0013191042000274196

Braun, D., Gable, R., y Kite, S. (2011). Situated in a Community of Practice: Leadership Preparation Practices to Support Leadership in K-8 Schools. The International Journal of Educational Leadership Preparation, 6(1), 1-17.

Burton, D., y Martens, R. (1986). Pinned by their own goals: An exploraty investigation into why kids drop-out of wrestling. Journal of Sport Psychology, 8, 183-197.

Cohn, P. J. (1990). An exploratory study on sources of stress and athlete burnout in youth golf. The Sport Psychologist, 2, 95-106.

Connelly, M., y Clandinin, J. (1995). Relatos de experiencia e investigación narrativa. En J. Larrosa (Ed.). Déjame que te cuente. Ensayos sobre narrativa y educación. Barcelona: Laertes.

Fiorese, L., Andrade, J. R., y Vieira, J. L. (2013). Perfeccionismo y nivel de cohesión de grupo de deportistas adultos de fútbol sala. Revista de Psicología del Deporte, 22 (2), 331-336.

Flett, G.L., y Hewitt, P.L. (2005). The perils of perfectionism in sports and exercise. Current Directions in Psychological Science, 14 (1), 14-18.

García-Fernández, J.M., Inglés, C.J., Vicent, M., Gonzálvez, C., Gómez-Núñez, M. I., y Poveda-Serra, P. (2016). Perfeccionismo durante la infancia y la adolescencia. Análisis bibliométrico y temático (2004-2014). Revista Iberoamericana de Psicología y Salud, 7, 79-88. doi: 10.1016/j.rips.2016.02.001

Goetz, J.P., y Lecompte, M.D. (1988). Características y orígenes de la etnografía educativa. Etnografía y diseño cualitativo en investigación educativa. Madrid: Morata.

Greblo, Z., Bari , R., y Ceci , E.S. (2016). Perfectionistic Strivings and Perfectionistic Concerns in Athletes: The Role of Peer Motivational Climate. Current Psychology, 35(3), 370-376.

Inglés, C.J., García-Fernández, J.M., Vicent, M., Gonzálvez, C. y Sanmartín, R. (2016). Profiles of Perfectionism and School Anxiety: A Review of the $2 \times 2$ Model of Dispositional Perfectionism in Child Population. Frontiers in Psychology, 7 (1403). doi: 10.3389/fpsyg.2016.01403

Malkinson, T.J., y He, C. (2014). Sports for Global Citizenship. IEEE Canada Internacional Humanitarian Tecnhology Conference, Canada.

Martínez-Navarro, A. (1993). Los valores de la Naturaleza en la Historia de la Educación desde la óptica de la Educación en el tiempo libre. Revista Complutense de Educación, 4(1), 119-143.

Martin, P. (2008). Outdoor adventure in promoting relationships with nature. Australian Journal of Outdoor Education, 8(1), 20-28, 2004.

Mollá-Serrano, M. (2007). La influencia de las actividades: Extraescolares en los Hábitos deportivos de los Escolares. Revista Internacional de Medicina y Ciencias de la Actividad Física y el Deporte, $7(27), 241-252$.

Murillo-Castro, L., Muñoz- Rojas, D., y Elizondo-Salazar, H. (2009). El campamento como estrategia metodológica para fortalecer las habilidades para la vida de los jóvenes estudiantes de Enfermería. Enfermería Actual en Costa Rica, 17, 1-16. 
Neely, K.C., y Holt, N. (2014). Parent's Perspectives on the Benefits of Sport Participation for Young Children. The Sport Psychologist, 28, 255-268.

Potter, J., y Hepburn, A. (2005). Qualitative interviews in psychology problems and possibilities. Qualitative Research in Psychology, 2, 1-27.

Pryor, A., Carpenter, C., y Townsend, M. (2005). Outdoor education and bush adventure therapy: A social-ecological approach to health and wellbeing. Australian journal of outdoor education, 9(1), 3-13.

Slade, P.D., y Owens, G.R. (1998). A dual process model of perfectionism based on reinforcement theory. Behavior Modification, 22 (3), 372-390.

Smith, L.M. (2015). Implementing a Multi-Sport Skills Camp for Girls Ages 8-11 with Autism Spectrum Disorder. (Thesis Submitted in Partial Fulfillment of the Requirements for the Degree of Master of Health Sciences). University of Ontario, California.

Stoeber, J., y Otto, K. (2006). Positive conceptions of perfectionism: Approaches, evidence, challenges. Personality and Social Psychology Review, 10, 295-319.

Stoeber, J., Stoll, 0., Salmi, 0., y Tiikkaja, J. (2009). Perfectionism and achievement goals in young Finnish ice-hockey players aspiring to make the Under-16 national team. Journal of Sports Sciences, 27, 85-94.

Stoeber, J. (2011). The dual nature of perfectionism in sports: Relationships with emotion, motivation, and performance. International Review of Sport and Exercise Psychology, 4(2), 128-145.

Stoeber, J. (2014). Perfectionism in Sport and Dance: A Double-Edged Sword. International Journal of Sport Psychology, 45, 385-394.doi:10.7352/IJSP 2014.45.385

Vicent, M., Inglés, C.J., Gonzálvez, C., Sanmartín, R., García-Fernández, J.M. (2016). Perfeccionismo socialmente prescrito y los cinco grandes rasgos de la personalidad en niños españoles. European Journal of Investigation in Health, Psychology and Education, 6(2), 107118.

Zurita-Pérez, R. (2009). Realización de actividades de aventura en el medio natural en el área de Educación Física. Innovación y Experiencias Educativas, 19, 1-9. 